\title{
SUMMABILITY OF A SEQUENCE OF FOURIER COEFFICIENTS BY A TRIANGULAR MATRIX TRANSFORMATION
}

H. P. DIKSHIT

1. Definitions and notations. Let $\sum_{n=0}^{\infty} v_{n}$ be a given infinite series with the sequence of partial sums $\left\{s_{n}\right\}$. We shall consider a sequence to sequence transformation of the type

$$
u_{n}=\sum_{k=0}^{\infty} d_{n k} s_{k}, \quad d_{n k}=0 \quad \text { for } k>n,
$$

in which the elements of the matrix $D=\left(\left(d_{n k}\right)\right)$ are real or complex constants. The sequence $\left\{u_{n}\right\}$ is called the sequence of $D$-means of $\left\{s_{n}\right\}$. If $\lim _{n \rightarrow \infty} u_{n}$ exists and is equal to $u$ then we say that the series $\sum v_{n}$ or the sequence $\left\{s_{n}\right\}$ is summable $D$ to the sum $u$.

Let $\left\{p_{n}\right\}$ be a sequence of constants, real or complex, and let us write $P_{n}=p_{0}+p_{1}+\cdots+p_{n} \neq 0, P_{-1}=p_{-1}=0$. Then the matrix $D$ defines a Nörlund matrix $\left(N, p_{n}\right)[6]$ if

$$
d_{n k}=p_{n-k} / P_{n}, \quad(n \geqq k \geqq 0) .
$$

The conditions for the regularity of the $\left(N, p_{n}\right)$ mean are

$$
\lim _{n \rightarrow \infty} p_{n} / P_{n}=0 \quad \text { and } \quad \sum_{k=0}^{n}\left|p_{k}\right|=O\left(\left|P_{n}\right|\right), \quad n \rightarrow \infty .
$$

In the special case in which

$$
p_{n}=\left(\begin{array}{c}
n+\alpha-1 \\
\alpha-1
\end{array}\right)=\frac{\Gamma(n+\alpha)}{\Gamma(n+1) \Gamma(\alpha)} \quad(\alpha>-1),
$$

the $\left(N, p_{n}\right)$ mean reduces to the familiar $(C, \alpha)$ mean. If $p_{n}=(n+1)^{-1}$ the $\left(N, p_{n}\right)$ mean is called the harmonic mean and is denoted by $(N, 1 /(n+1))$.

The product of the $(C, 1)$ matrix with a $\left(N, p_{n}\right)$ matrix defines the matrix $(C, 1) \cdot\left(N, p_{n}\right)$. Thus the $(C, 1) \cdot\left(N, p_{n}\right)$ matrix is given by

$$
d_{n k}=\frac{1}{n+1} \sum_{r=k}^{n} p_{r-k} / P_{r} \quad(0 \leqq k \leqq n),
$$

and the corresponding mean of $\left\{s_{n}\right\}$ is

Received by the editors April 2, 1968. 


$$
u_{n}=\sum_{k=0}^{n} \frac{1}{n+1} \sum_{r=k}^{n} p_{r-k} s_{k} / P_{r}=\frac{1}{n+1} \sum_{r=0}^{n} \frac{1}{P_{r}} \sum_{k=0}^{r} p_{r-k} s_{k} .
$$

Similarly, one defines the $\left(N, p_{n}\right) \cdot(C, 1)$ summability.

Silverman has shown in [7] that a necessary and sufficient condition for a $\left(N, p_{n}\right)$ matrix to be permutable with the $(C, 1)$ matrix is that it be a Cesàro matrix. This implies inter alia that $(C, 1) \cdot\left(N, p_{n}\right)$ $\neq\left(N, p_{n}\right) \cdot(C, 1)$ except when $\left\{p_{n}\right\}$ is defined by (1.4).

Let $f(t)$ be integrable $L$ in $(-\pi, \pi)$ and periodic with period $2 \pi$, and let

$$
f(t) \sim \frac{1}{2} a_{0}+\sum_{n=1}^{\infty}\left(a_{n} \cos n t+b_{n} \sin n t\right) .
$$

Then the conjugate series of (1.7) at $t=x$ is

$$
\sum_{n=1}^{\infty}\left(b_{n} \cos n x-a_{n} \sin n x\right)=\sum_{n=1}^{\infty} B_{n}(x) .
$$

We write throughout:

$$
\begin{aligned}
\psi(t) & =f(x+t)-f(x-t)-l, \text { where } l \text { is a finite number. } \\
\Psi(t) & =\int_{0}^{t}|\psi(u)| d u . \\
S_{n} & =\frac{1}{P_{n}} \sum_{\nu=0}^{n} \frac{P_{\nu}}{\nu+1} ; \quad \Delta_{n} \lambda_{n}=\lambda_{n}-\lambda_{n+1} .
\end{aligned}
$$

$\tau=[1 / t], P_{\tau}=P(1 / t)$, where $\tau$ denotes the greatest integer not greater than $1 / t$.

$K$ denotes a positive constant not necessarily the same at each occurrence.

2. Introduction. Concerning the $(C, 1)$ summability and the $(N, 1 /(n+1)) \cdot(C, 1)$ summability of the sequence $\left\{n B_{n}(x)\right\}$, Mohanty and Nanda [5] and Varshney [8] have proved the following results.

TheOREM A. If $\psi(t)=o\left(\{\log (1 / t)\}^{-1}\right)$ as $t \rightarrow 0$ and

$$
a_{n}=O\left(n^{-\delta}\right), \quad b_{n}=O\left(n^{-\delta}\right), \quad 0<\delta<1,
$$

then the sequence $\left\{n B_{n}(x)\right\}$ is summable $(C, 1)$ to the value $l / \pi$.

Theorem B. If 


$$
\Psi(t)=o\left\{t\left(\log \frac{1}{t}\right)^{-1}\right\}, \quad t \rightarrow 0,
$$

then the sequence $\left\{n B_{n}(x)\right\}$ is summable $(N, 1 /(n+1)) \cdot(C, 1)$ to $l / \pi$.

It is apparent from Silverman's result [7] that the summability $(C, 1) \cdot(N, 1 /(n+1))$ is different from the summability $(N, 1 /(n+1))$ $\cdot(C, 1)$. However, it may be observed that the summability $(N, 1 /(n+1))$ implies the $(C, \delta)$ summability for any $\delta>0$ and, therefore, from a well-known result concerning the $(C)$ summability, it follows that both $(C, 1) \cdot\left(N, p_{n}\right)$ as well as $(N, 1 /(n+1)) \cdot(C, 1)$ imply the $(C, 1+\delta)$ summability, for some suitable $\left\{p_{n}\right\}$. In view of this it is natural to expect that the hypothesis (2.2) of Theorem B may lead to the summability $(C, 1) \cdot(N, 1 /(n+1))$ of the sequence $\left\{n B_{n}(x)\right\}$. That this is indeed true is demonstrated by the special case $p_{n}$ $=(n+1)^{-1}$ of our Theorem 1 stated in the next section.

It is known that if $\psi(t)=o(1), t \rightarrow 0$, then the sequence $\left\{n B_{n}(x)\right\}$ is summable $(C, r), r>1$, to the value $l / \pi$ and that it is a best possible result in the sense that it breaks down for $r=1[9, \mathrm{p} .62]$. This result follows as a corollary from our Theorem 2 when one observes that the sequence $\left\{p_{n}\right\}$ defined by (1.4) satisfies the hypotheses of Theorem 2 if $0<\alpha<1$.

3. We prove the following.

THEOREM 1. Let $\left\{p_{n}\right\}$ be a nonnegative, monotonic nonincreasing sequence such that $\log n=O\left(P_{n}\right), n \rightarrow \infty$. Then, if

$$
\Psi(t)=o\left(t / P_{\tau}\right), \quad t \rightarrow 0,
$$

the sequence $\left\{n B_{n}(x)\right\}$ is summable $(C, 1) \cdot\left(N, p_{n}\right)$ to the value $l / \pi$.

Theorem 2. If $\psi(t)=o(1), t \rightarrow 0$, and $\left\{p_{n}\right\}$ is a nonnegative, monotonic nonincreasing sequence such that $S_{n}=O(1)$, then the sequence $\left\{n B_{n}(x)\right\}$ is summable $(C, 1) \cdot\left(N, p_{n}\right)$ to the value $l / \pi$.

4. We need the following lemmas for the proof of our theorems.

LEMмA 1. If $\left\{p_{n}\right\}$ is a positive sequence such that $(n+1) p_{n} \leqq K P_{n}$ and $S_{n}=O(1)$, then $P_{n} \rightarrow \infty$ as $n \rightarrow \infty$.

Proof. As pointed out by the present author in [1], the hypotheses of Lemma 1 imply that

$$
\sum_{k=n+1}^{\infty} \frac{1}{(k+1) P_{k-1}}=O\left(\frac{1}{P_{n}}\right) \quad(n=1,2,3, \cdots),
$$


and therefore $P_{n} \rightarrow \infty$ as $n \rightarrow \infty$. It may be remarked that with a slight modification in the reasoning given by the present author in [1] it is possible to avoid the hypothesis $(n+1) p_{n} \leqq K P_{n}$.

LEMMA 2. If $\left\{p_{n}\right\}$ is nonnegative and nonincreasing, then for $0 \leqq a$ $\leqq b \leqq \infty$ and $0 \leqq t \leqq \pi$,

$$
\left|\sum_{k=a}^{b} p_{k} \exp i k t\right| \leqq K P_{r} .
$$

Lemma 2 may be proved by following the technique of proof of Lemma 5.11 in McFadden [4].

LEMMA 3. If $\left\{p_{n}\right\}$ is nonnegative and nonincreasing, then as $n \rightarrow \infty$

$$
\begin{aligned}
\sum_{\nu=0}^{n} \frac{1}{P_{\nu}} \sum_{k=0}^{\nu}(\nu-k) p_{k} \exp i(\nu & -k) t \\
& =O\left(t^{-2}\right)+O\left(t^{-1} P_{\tau} \sum_{\nu=\tau}^{n} \frac{1}{P_{\nu}}\right)+O\left(\frac{n t^{-1} P_{\tau}}{P_{n+1}}\right)
\end{aligned}
$$

uniformly in $0<t \leqq \pi$.

Proof. Applying Abel's transformation we get

$$
\begin{aligned}
\sum_{\nu=k}^{n}(\nu-k) \frac{1}{P_{\nu}} \exp i(\nu-k) t & \\
= & \sum_{\nu=k}^{n} \Delta_{\nu}\left(\frac{\nu-k}{P_{\nu}}\right) \sum_{\mu=k}^{\nu} \exp i(\mu-k) t+\frac{n-k+1}{P_{n+1}} \sum_{\mu=k}^{n} \exp i(\mu-k) t \\
= & (1-\exp i t)^{-1}\left[\sum_{\nu=k}^{n} \Delta_{\nu}\left(\frac{\nu-k}{P_{\nu}}\right)\{1-\exp i(\nu-k+1) t\}\right. \\
& \left.\quad+\frac{n-k+1}{P_{n+1}}\{1-\exp i(n-k+1) t\}\right] \\
=(1-\exp i t)^{-1}\left[-\sum_{\nu=k}^{n} \frac{p_{\nu+1}}{P_{\nu} P_{\nu+1}}(\nu-k) \exp i(\nu-k+1) t\right. & \quad+\frac{1}{\sum_{\nu=k}^{n} \frac{1}{P_{\nu+1}} \exp i(\nu-k+1) t} \\
& \left.-\frac{n-k+1}{P_{n+1}} \exp i(n-k+1) t\right] .
\end{aligned}
$$

Changing the order of summation of the inner sums, thus we have 


$$
\begin{aligned}
\sum & \equiv\left|\sum_{\nu=0}^{n} \frac{1}{P_{\nu}} \sum_{k=0}^{\nu}(\nu-k) p_{k} \exp i(\nu-k) t\right| \\
& =\left|\sum_{k=0}^{n} p_{k} \sum_{\nu=k}^{n} \frac{(\nu-k)}{P_{\nu}} \exp i(\nu-k) t\right| \\
& \leqq K t^{-1}\left[\left|\sum_{k=0}^{n} p_{k} \sum_{\nu=k}^{n} \frac{p_{\nu+1}}{P_{\nu} P_{\nu+1}}(\nu-k) \exp i(\nu-k+1) t\right|\right. \\
& \quad+\left|\sum_{k=0}^{n} p_{k} \sum_{\nu=k}^{n} \frac{1}{P_{\nu+1}} \exp i(\nu-k+1) t\right| \\
& \left.\quad+\frac{1}{P_{n+1}}\left|\sum_{k=0}^{n} p_{k}(n-k+1) \exp i(n-k+1) t\right|\right] \\
& =\sum_{1}+\sum_{2}+\sum_{3}, \quad \text { say. }
\end{aligned}
$$

Again, by a change of order of summation, we have

$$
\begin{aligned}
\sum_{1} \leqq & K t^{-1} \sum_{\nu=0}^{n} \frac{p_{\nu+1}}{P_{\nu} P_{\nu+1}}\left|\sum_{k=0}^{p} p_{k}(\nu-k) \exp i(\nu-k+1) t\right| \\
\leqq & K t^{-1} \sum_{\nu=0}^{\tau-1} \frac{\nu p_{\nu+1}}{P_{\nu} P_{\nu+1}} \sum_{k=0}^{n} p_{k} \\
& +K t^{-1} \sum_{\nu=r}^{n} \frac{\nu p_{\nu+1}}{P_{\nu} P_{\nu+1}} \max _{0 \leqq p \leqq \nu}\left|\sum_{k=0}^{p} p_{k} \exp i(\nu-k+1) t\right|
\end{aligned}
$$

(by Abel's Lemma, the first sum is taken as zero if $\tau=0$ )

$$
\leqq K t^{-2}+K t^{-1} P_{\tau} \sum_{\nu=\tau}^{n} \frac{1}{P_{\nu}},
$$

by virtue of Lemma 2 and the fact that $(\nu+1) p_{\nu} \leqq P_{\nu}$.

Similarly,

by Lemma 2 .

$$
\begin{aligned}
\sum_{2} & \leqq K t^{-1} \sum_{\nu=0}^{n} \frac{1}{P_{\nu+1}}\left|\sum_{k=0}^{\nu} p_{k} \exp i(\nu-k+1) t\right| \\
& \leqq K t^{-2}+K t^{-1} P_{\tau} \sum_{\nu=r}^{n} \frac{1}{P_{\nu}}
\end{aligned}
$$

Finally, by Lemma 2 and Abel's Lemma, we have

$$
\sum_{3} \leqq K t^{-1} n P_{\tau} \frac{1}{P_{n+1}},
$$

which completes the proof of the lemma. 
5. Proof of Theorem 1. We have

$$
\begin{aligned}
n B_{n}(x) & =\frac{1}{\pi} \int_{0}^{\pi}\{f(x+t)-f(x-t)\} n \sin n t d t \\
& =\frac{1}{\pi} \int_{0}^{\pi} \psi(t) n \sin n t d t+\frac{l}{\pi}\left\{1-(-1)^{n}\right\} .
\end{aligned}
$$

Therefore, if $\left\{t_{n}(x)\right\}$ denotes the $\left(N, p_{n}\right)$ mean of $\left\{n B_{n}(x)\right\}$, then

$$
\begin{aligned}
t_{n}(x) & -\frac{l}{\pi}=\frac{1}{\pi P_{n}} \int_{0}^{\pi} \psi(t)\left(\sum_{k=0}^{n} p_{n-k} k \sin k t\right) d t \\
& -\frac{l}{\pi P_{n}} \sum_{k=0}^{n} p_{n-k}(-1)^{k} .
\end{aligned}
$$

Since $\left\{p_{n}\right\}$ is nonnegative and nonincreasing and $P_{n} \rightarrow \infty$ as $n \rightarrow \infty$, we have by an application of Abel's Lemma

$$
t_{n}(x)-\frac{l}{\pi}=o(1)+\frac{1}{\pi P_{n}} \int_{0}^{\pi} \psi(t)\left(\sum_{k=0}^{n} p_{n-k} k \sin k t\right) d t .
$$

Denoting the $(C, 1) \cdot\left(N, p_{n}\right)$ mean of $\left\{n B_{n}(x)\right\}$ by $t_{n}^{1}(x)$, we write

$$
t_{n}^{1}(x)-\frac{l}{\pi}=o(1)+\frac{1}{\pi(n+1)} \int_{0}^{\pi} \psi(t)\left\{\sum_{v=0}^{n} \frac{1}{P_{\nu}} \sum_{k=0}^{n} p_{v-k} k \sin k t\right\} d t
$$

by the regularity of the $(C, 1)$ mean. Thus, in order to prove the theorem, it is sufficient to show that as $n \rightarrow \infty$

$$
I \equiv \frac{1}{n+1} \int_{0}^{\pi} \psi(t) g(n, t) d t=o(1)
$$

where

$$
g(n, t)=\sum_{\nu=0}^{n} \frac{1}{P_{\nu}} \sum_{k=0}^{\nu} p_{k}(\nu-k) \sin (\nu-k) t
$$

For a sufficiently small $\delta$, such that $0<\delta \leqq \pi$, let us write

$$
\begin{aligned}
I & =\frac{1}{n+1}\left(\int_{0}^{n^{-1}}+\int_{n^{-1}}^{\delta}+\int_{\delta}^{\pi}\right) \psi(t) g(n, t) d t \\
& =I_{1}+I_{2}+I_{3}, \text { say. }
\end{aligned}
$$


Since

$$
|g(n, t)| \leqq \sum_{\nu=0}^{n} \frac{1}{P_{\nu}} \sum_{k=0}^{\prime} p_{k}(\nu-k) \leqq K n^{2},
$$

we have by the hypothesis (3.1)

$$
I_{1}=o\left(n \int_{0}^{1 / n}|\psi(t)| d t\right)=o\left(1 / P_{n}\right)=\dot{o}(1) \quad \text { as } n \rightarrow \infty .
$$

By virtue of Lemma 3 for the interval $\delta \leqq t \leqq \pi$, we have

$$
\frac{1}{n} g(n, t)=O\left(\frac{1}{n}\right)+O\left(\frac{1}{n} \sum_{\nu=0}^{n} \frac{1}{P_{\nu}}\right)+O\left(\frac{1}{P_{n}}\right)=o(1),
$$

$$
n \rightarrow \infty
$$

since the $(C, 1)$ mean is regular and $P_{n} \rightarrow \infty$ as $n \rightarrow \infty$. Thus, as $n \rightarrow \infty$

$$
I_{3}=\frac{1}{n} \int_{\delta}^{\pi} \psi(t) g(n, t) d t=o(1) .
$$

Finally, to prove that $I_{2}=o(1), n \rightarrow \infty$, we observe that by Lemma 3

$$
\begin{aligned}
I_{2}= & O\left(\frac{1}{n} \int_{n^{-1}}^{\delta} t^{-2}|\psi(t)| d t\right) \\
& +O\left(\frac{1}{n} \int_{n^{-1}}^{\delta} \frac{|\psi(t)|}{t}\left\{P_{\tau} \sum_{v=\tau}^{n} \frac{1}{P_{v}}\right\} d t\right) \\
& +O\left(\frac{1}{P_{n}} \int_{n^{-1}}^{\delta} \frac{|\psi(t)|}{t} P_{\tau} d t\right) \\
= & O\left(I_{21}\right)+O\left(I_{22}\right)+O\left(I_{23}\right), \text { say. }
\end{aligned}
$$

Integrating by parts, we have as $n \rightarrow \infty$

$$
\begin{aligned}
I_{21} & =\frac{1}{n}\left[t^{-2} \Psi(t)\right]_{n}^{\delta}+\frac{2}{n} \int_{n^{-1}}^{\delta} \Psi(t) t^{-3} d t \\
& =o\left(1 / P_{n}\right)+o(1)+o\left(\frac{1}{n} \int_{n-1}^{\delta} t^{-2} \frac{1}{P_{\tau}} d t\right) \\
& =o(1)+o\left(\frac{1}{n} \int_{n-1}^{\delta} t^{-2} d t\right)=o(1),
\end{aligned}
$$

by the hypothesis (3.1). 
Let $V(n, r)=P_{r} \sum_{k=r}^{n}\left(1 / P_{k}\right)$ and $n^{-1}<m^{-1} \leqq \delta<(m-1)^{-1}$, then we write $^{1}$

$$
\begin{aligned}
I_{22} & =\frac{1}{n}\left(\sum_{r=m}^{n-1} \int_{(r+1)^{-1}+0}^{r^{-1}}+\int_{m^{-1}}^{\delta}\right) \frac{|\psi(t)|}{t} V(n, \tau) d t \\
& =I_{221}+I_{222}, \text { say. }
\end{aligned}
$$

In the interval $(r+1)^{-1}<t \leqq r^{-1}$, we have $V(n, \tau)=V(n, r)$, and therefore integrating by parts we get

$$
\begin{aligned}
& \int_{(r+1)^{-1}+0}^{r^{-1}} V(n, \tau) \frac{|\psi(t)|}{t} d t \\
& \quad=V(n, r)\left[\frac{\Psi(t)}{t}\right]_{(r+1)-1}^{r^{-1}}+\int_{(r+1)^{-1}+0}^{r^{-1}} V(n, \tau) \frac{\Psi(t)}{t^{2}} d t .
\end{aligned}
$$

Further, by (3.1) we have

$$
\begin{aligned}
\sum_{r=m}^{n-1} V(n, r) & \left\{r \Psi\left(\frac{1}{r}\right)-(r+1) \Psi\left(\frac{1}{r+1}\right)\right\} \\
= & \sum_{r=m}^{n-1} \Delta_{r}\left\{r \Psi\left(\frac{1}{r}\right) V(n, r)\right\} \\
& -\sum_{r=m}^{n-1}(r+1) \Psi\left(\frac{1}{r+1}\right) \Delta_{r} V(n, r) \\
= & m \Psi\left(\frac{1}{m}\right) V(n, m)-n V(n, n) \Psi\left(\frac{1}{n}\right) \\
& +o\left[\sum_{r=m}^{n-1} \frac{p_{r+1}}{P_{r+1}} \sum_{k=r}^{n} \frac{1}{P_{k}}-\sum_{r=m}^{n-1} \frac{1}{P_{r}}\right] \\
= & o\left(\sum_{r=m}^{n} \frac{1}{P_{v}}\right)+o(1)+o\left[\sum_{k=m}^{n} \frac{1}{P_{k}} \sum_{r=m}^{k} \frac{P_{r+1}}{P_{r+1}}\right]=o(n),
\end{aligned}
$$

since $(n+1) p_{n} \leqq P_{n}$ and $\log n=O\left(P_{n}\right)$.

Combining (5.11) and (5.12), we obtain

$$
I_{221}=o(1)+\int_{n^{-1}}^{m^{-1}} \frac{\Psi(t)}{t^{2}} \frac{1}{n} V(n, \tau) d t .
$$

Similarly, it follows that

\footnotetext{
${ }^{1}$ Here $m$ is an integer.
} 


$$
I_{222}=o(1)+\int_{m^{-1}}^{\delta} \frac{\Psi(t)}{t^{2}} \frac{1}{n} V(n, \tau) d t
$$

since $(1 /(n+1)) \sum_{k=m}^{n}\left(1 / P_{k}\right)=o(1)$ by virtue of the regularity of the $(C, 1)$ mean and the fact that $P_{n} \rightarrow \infty$ as $n \rightarrow \infty$.

Thus, we have from (5.13) and (5.14)

$$
\begin{aligned}
I_{22} & =o(1)+\frac{1}{n} \int_{n^{-1}}^{\delta} t^{-2} \Psi(t) V(n, \tau) d t \\
& =o\left[\frac{1}{n} \int_{n^{-1}}^{\delta} \frac{1}{t}\left(\sum_{r=r}^{n} \frac{1}{P_{r}}\right) d t\right] \text { by }(3.1) \\
& =o\left[\frac{1}{n} \int_{\delta}^{n-1} \frac{1}{y}\left(\sum_{r=[y]}^{n} \frac{1}{P_{r}}\right) d y\right]=o(1), \quad n \rightarrow \infty,
\end{aligned}
$$

since

$$
\sum_{k=1}^{n} \frac{1}{k} \sum_{r=k}^{n} \frac{1}{P_{r}}=\sum_{r=1}^{n} \frac{1}{P_{r}} \sum_{k=1}^{r} \frac{1}{k}=O(n)
$$

by the hypothesis $\log n=O\left(P_{n}\right)$.

Following a technique parallel to that used in the proof of $I_{22}=o(1)$, we observe that

$$
\begin{aligned}
I_{23}=o(1)+\frac{1}{P_{n}} \int_{n^{-1}}^{\delta} t^{-2} \Psi(t) P_{\tau} d t=o\left(\frac{1}{P_{n}} \int_{n^{-1}}^{\delta} t^{-1} d t\right)= & o(1), \\
& n \rightarrow \infty,
\end{aligned}
$$

by (3.1) and that $\log n=O\left(P_{n}\right)$.

Combining (5.9), (5.15) and (5.16) we get $I_{2}=o(1), n \rightarrow \infty$, which together with (5.5) and (5.7) implies (5.1).

This completes the proof of the theorem.

6. Proof of Theorem 2. As in the proof of Theorem 1, in order to prove Theorem 2 it is sufficient to show that as $n \rightarrow \infty$

$$
I_{k}=o(1) \quad(k=1,2,3),
$$

under the hypotheses of Theorem 2 .

Since by (5.4), (1/n) $g(n, t)=O(n)$ and $\psi(t)=o(1), t \rightarrow 0$, we have as $n \rightarrow \infty$

$$
I_{1}=o(1) \text {. }
$$

From (5.6) it follows that as $n \rightarrow \infty$ 


$$
I_{3}=o(1)
$$

By Lemma 3, we have

$$
\begin{aligned}
\frac{1}{n} \int_{n^{-1}}^{\delta}|g(n, t)| d t \leqq \frac{K}{n} \int_{n^{-1}}^{\delta} t^{-2} d t & \\
& +\frac{K}{n} \int_{n^{-1}}^{\delta} \frac{P(1 / t)}{t}\left\{\sum_{\nu=\tau}^{n} \frac{1}{P_{\nu}}\right\} d t+\frac{K}{P_{n}} \int_{n^{-1}}^{\delta} \frac{P(1 / t)}{t} d t \\
& \leqq K+\frac{K}{n} \int_{\delta^{-1}}^{n} \frac{P(y)}{y}\left\{\sum_{\nu=[y]}^{n} \frac{1}{P_{\nu}}\right\} d y+\frac{K}{P_{n}} \int_{\delta^{-1}}^{n} \frac{P(y)}{y} d y \\
\leqq & K+\frac{K}{n} \int_{\delta^{-1}}^{n} \frac{P(y)}{y}\left\{\sum_{\nu=[y]}^{n} \frac{1}{P_{\nu}}\right\} d y \leqq K,
\end{aligned}
$$

since $S_{n}=O(1)$ and

$$
\frac{1}{n} \sum_{k=1}^{n} \frac{P_{k}}{k} \sum_{\nu=k}^{n} \frac{1}{P_{\nu}}=\frac{1}{n} \sum_{\nu=1}^{n} \frac{1}{P_{\nu}} \sum_{k=1}^{\nu} \frac{P_{k}}{k} \leqq \frac{1}{n} \sum_{\nu=1}^{n} S_{\nu} \leqq K .
$$

Finally, since $\psi(t)=o(1), t \rightarrow 0$, it follows from (6.4) that

$$
I_{2}=\frac{1}{n} \int_{n^{-1}}^{\delta} \psi(t) g(n, t) d t=o(1) \quad \text { as } n \rightarrow \infty .
$$

Combining (6.2), (6.3) and (6.5), we prove (6.1), which completes the proof of Theorem 2 .

7. It may be remarked that the hypothesis $S_{n}=O(1)$ of our Theorem 2 has its origin in [2], where its intrinsic character has been discussed by Hille and Tamarkin.

By making use of a Tauberian result due to Iyengar [3], Varshney has pointed out in [8] that under the condition (2.1) of Theorem A $(N, 1 /(n+1)) \cdot(C, 1)$ summability of $\left\{n B_{n}(x)\right\}$ implies its $(C, 1)$ summability. In view of this, Theorem $A$ and our Theorem 1 suggest the open question of $(C, 1) \cdot(N, 1 /(n+1))$ summability of $\left\{n B_{n}(x)\right\}$ implying its $(C, 1)$ summability under the condition $(2.1)$.

\section{REFERENCES}

1. H. P. Dikshit, Absolute summability of Fourier series by Nörlund means, Math. Z. 102 (1967), 166-170.

2. E. Hille and J. D. Tamarkin, On the summability of Fourier series. I, Trans. Amer. Math. Soc. 34 (1932), 757-783.

3. K. S. K. Iyengar, A Tauberian theorem and its application to convergence of Fourier series, Proc. Indian Acad. Sci. Sect. A 18 (1943), 81-87. 
4. L. McFadden, Absolute Nörlund summability, Duke Math. J. 9 (1942), 168-207.

5. R. Mohanty and M. Nanda, On the behavior of Fourier coefficients, Proc. Amer. Math. Soc. 5 (1954), 79-84.

6. N. E. Nörlund, Sur une application des fonctions permutables, Lunds Univ. Årsskr. Avd. 216 (1919), no. 3.

7. S. L. Silverman, Products of Nörlund transformation, Bull. Amer. Math. Soc. 43 (1937), 95-101.

8. O. P. Varshney, On a sequence of Fourier coefficients, Proc. Amer. Math. Soc. 10 (1949), 790-795.

9. A. Zygmund, Trigonometric series, Monogr. Mat. Bd. 5, Warsaw, 1935.

University of Allahabad, Allahabad, India 